\title{
Formulating spread of species with habitat dependent growth and dispersal in heterogeneous landscapes
}

\author{
Andriamihaja Ramanantoanina ${ }^{\mathrm{a}, \mathrm{b}, *}$, Cang Hui ${ }^{\mathrm{a}, \mathrm{b}}$ \\ ${ }^{a}$ Centre for Invasion Biology, Department of Mathematical Sciences, Stellenbosch University, Matieland 7602, South Africa \\ ${ }^{\mathrm{b}}$ Mathematical and Physical Biosciences, African Institute for Mathematical Sciences, Cape Town 7945, South Africa
}

\section{A R T I C L E I N F O}

\section{Article history:}

Received 26 August 2015

Revised 31 December 2015

Accepted 28 February 2016

Available online 8 March 2016

\section{Keywords:}

Biological invasion

Range expansion

Boom-and-bust

Lag phase

Habitat heterogeneity

Dispersal strategy

\begin{abstract}
A B S T R A C T
Habitat heterogeneity can have profound effects on the spreading dynamics of invasive species. Using integro-difference equations, we investigate the spreading dynamics in a one-dimensional heterogeneous landscape comprising alternating favourable and unfavourable habitat patches or randomly generated habitat patches with given spatial autocorrelation. We assume that population growth and dispersal (including emigration probability and dispersal distance) are dependent on habitat quality. We derived an approximation of the rate of spread in such heterogeneous landscapes, suggesting the sensitivity of spread to the periodic length of the alternating favourable and unfavourable patches, as well as their spatial autocorrelation. A dispersal-limited population tends to spread faster in landscapes with shorter periodic length. The spreading dynamics in a heterogeneous landscape was found to be not only dependent on the availability of favourable habitats, but also the dispersal strategy. Estimates of time lag before detection and the condition for boom-and-bust spreading dynamics were explained. Furthermore, rates of spread in heterogeneous landscapes and corresponding homogeneous landscapes were compared, using weighted sums of vital rates.
\end{abstract}

(c) 2016 Elsevier Inc. All rights reserved.

\section{Introduction}

Landscape heterogeneity can affect the behaviour of biological invasions at different stages, especially when established species start to become invasive and spread into heterogeneous landscapes [1-3]. Empirical investigations have suggested that the spatial heterogeneity of landscapes can influence the rate of spread of invasive species [4] as demography and dispersal are both context based (i.e. sensitive to spatial heterogeneity) [1,5]. While many robust estimates of the asymptotic rate of spread on homogeneous landscapes have been formulated [6], the effects of spatial heterogeneity on the spreading dynamics of species with habitat sensitive demography and dispersal demand more attention $[7,8]$.

Invasion dynamics in heterogeneous landscapes has long been theoretically explored using continuous time frameworks such as partial differential equations. In particular, Shigesada et al. [9] simulate spatial heterogeneity by alternating homogeneously favourable and unfavourable habitat patches on an infinite onedimensional environment, with the growth rate and diffusion co-

\footnotetext{
* Corresponding author at: Mathematical and Physical Biosciences, African Institute for Mathematical Sciences, Cape Town 7945, South Africa. Tel.: +27 725569814.

E-mail address: mihataims@gmail.com, ar@aims.ac.za (A. Ramanantoanina).
}

efficient assumed as periodic step functions of locations. It emphasises the effect of the lengths of periodically alternating favourable and unfavourable patches on the rate of spread. In contrast, Kinezaki et al. [10] consider the effect of spatial heterogeneity by allowing vital rates to vary sinusoidally in space, representing a continuous change in habitat quality. It emphasises the role of both the amplitude and periodic length of habitat heterogeneity on the rate of spread. In both models, when the periodic length of alternating habitat quality is short, the initial population will propagate from the introduction point into periodic travelling waves, with the rate of spread being $c=2 \sqrt{\langle r\rangle_{A}\langle D\rangle_{H}}$, where $\langle r\rangle_{A}$ and $\langle D\rangle_{H}$ denote the spatial arithmetic mean of the growth rate and the spatial harmonic mean of the diffusion coefficient, respectively.

When the focal species does not follow a diffusion-type movement, integrodifference equations (IDEs) are commonly used for modelling the spatiotemporal dynamics of biological invasions [11]. For instance, Kawasaki and Shigesada [7] have examined the spreading dynamics with an exponentially damping (Laplace) dispersal kernel in a patchy landscape with alternating favourable and unfavourable patches, while assuming that dispersal is insensitive to habitat heterogeneity. They found that the presence of unfavourable patches can dramatically reduce the rate of spread, although the population can always spread with wide enough favourable habitats. Dewhirst and Lutscher [8] expanded this 
model by considering habitat dependent dispersal behaviours, with individuals from unfavourable habitats dispersing farther in an attempt to find favourable habitats. These works put the emphasis on the existence of a minimum proportion of favourable habitats for successful invasions and spread. Gilbert et al. [12] further consider the effects of the landscape periods on the spread of a structured population.

We here formulate the spread of a species with nonoverlapping generations in a heterogeneous patchy landscape as defined by Shigesada et al. [9]. Besides assuming a habitatdependent population growth, dispersal behaviours are further affected by habitat quality in the following two ways. First, the dispersal distance of migrating individuals is dependent on the habitat quality, with individuals from unfavourable locations dispersing farther for locating favourable habitats $[13,14]$. Second, only a fraction of the local population emigrates (defined as emigration probability) while others remain sedentary - the number being dependent on habitat quality. We also perform numerical simulations to investigate the instantaneous rate of spread, and derive an estimate for the asymptotic rate of spread in randomly generated patchy landscapes with different levels of spatial autocorrelation.

\section{Model}

We consider a population with non-overlapping generations undergoing growth and dispersal at separate times, using integrodifference equations (IDEs). With the vital rates affected by spatial heterogeneity, we have the following IDE model,

$$
\begin{aligned}
u(x, t+1)= & \int[d(y) k(x-y, y)+(1-d(y)) \delta(x-y)] \\
& \times g(u(y, t), y) d y
\end{aligned}
$$

where $u(x, t)$ denotes the population size at location $x$ and time $t$. The function $g$ gives the population growth (more specifically, fecundity in species with non-overlapping generations). It is a nonnegative function satisfying density dependent recruitment, $g(u$, $x) \leq R(x)$ with $R(x)=\partial g /\left.\partial u\right|_{u=0}$ being the intrinsic growth rate at location $x$. In the Ricker (1954) model, we have $g(u(x, t), x)=$ $u e^{r(x)-u(x, t)}$ and $R(x)=e^{r(x)}$.

We considered two factors in formulating the habitat dependent dispersal strategy [14,15]. First, the dispersal kernel $k$ in Eq. (1) gives the probability distribution that an individual from location $y$ disperses to location $x$. The dispersal distance effectuated by an individual during a dispersal event can be influenced by the habitat quality $[13,14]$. That is, $k(x-y, y)$ not only depends on the distance between location $x$ and $y$ but also the habitat quality of the originating location $y$. For instance, a Gaussian dispersal kernel is thus

$k(x-y, y)=\left(1 / \sqrt{2 \pi \sigma^{2}(y)}\right) \exp \left(-(x-y)^{2} /\left(2 \sigma^{2}(y)\right)\right)$,

and a Laplace dispersal kernel

$k(x-y, y)=\left(1 / \sqrt{2 \sigma^{2}(y)}\right) \exp \left(-\sqrt{2}|x-y| / \sqrt{2 \sigma^{2}(y)}\right)$.

Second, spatial heterogeneity can also influence the probability, $d(y)$, of an individual emigrating from its original location $y$ to other patches, often following a 'good-stay, bad-disperse' rule [5]. Therefore, $1-d(y)$ gives the proportion of individuals remaining sedentary, with $\delta(x-y)$ in Eq. (1) being 1 if $x=y$ and 0 otherwise.

Here we focused on periodically alternating habitats of favourable and unfavourable patches, with lengths $L_{1}$ and $L_{2}$, respectively $[7,9]$. The habitat was laid out with a periodic length of $L\left(=L_{1}+L_{2}\right)$ and a proportion of $p\left(=L_{1} / L_{2}\right)$ favourable habitats in the landscape. The intrinsic growth rate $R(x)$ is given by $R_{1}(>1$ to ensure population growing) in favourable habitats and $R_{2}(>0)$ in unfavourable habitats. Similarly, we also defined the emigration probability, $d(x)$, being $d_{1}$ and $d_{2}$, and the variation of dispersal
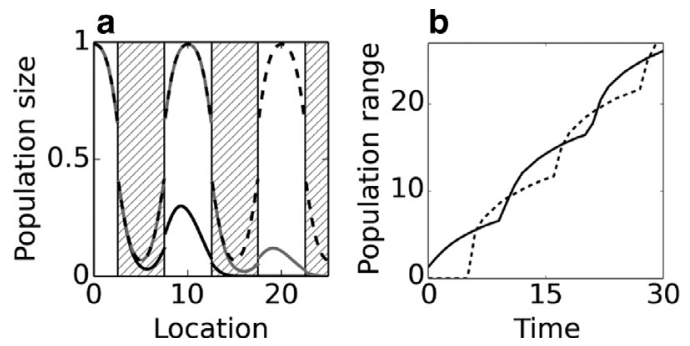

Fig. 1. (A) Travelling periodic waves in a heterogeneous landscape. Dashed black line represents the periodic steady state of the model. Solid black and grey lines indicate the population size at time $t=15$ and $t=25$ respectively. Hatched region indicate unfavourable patches. (B) Population range front for the initial population introduced in favourable (solid line) and unfavourable (dashed line) locations. While the former started to spread immediately, the population introduced into an unfavourable habitat experienced a lag between the introduction and detected spread. Parameter values are $p=0.5, R_{1}=e, R_{2}=e^{-0.5}, d_{1}=0.75, d_{2}=1, \sigma_{1}^{2}=1, \sigma_{2}^{2}=1$.

kernel, $\sigma^{2}(x)$, being $\sigma_{1}^{2}$ and $\sigma_{2}^{2}$ in favourable and unfavourable habitats, respectively.

To study the dynamics of the above IDE model, we first investigate its non-trivial steady states by replacing $u(x, t+1)$ and $u(y, t)$ in Eq. (1) with $v(x)$ and $v(y)$, and numerically solving the equation using the routine optimize.fsolve in the Python library SciPy [16]. To investigate the spreading dynamics, we ran the model for 100 generations and calculated the population range at time $t$ as $x^{*}(t)=\max \left\{x ; u(x, t) \geq u^{*}\right\}$ for a threshold of detection $u *$. The corresponding instantaneous and average rate of spread can be given $\operatorname{asc}_{I}(t)=x^{*}(t+1)-x^{*}(t)$ and $c_{A}(t)=x^{*}(t) / t$. The time lag before range expansion is defined as the first time when the population was detected after its introduction, $T\left(u^{*}\right)=\min \left\{t ; x^{*}(t)>\right.$ $0\}$. The spreading dynamics was also compared with the dynamics in homogenous landscape with normalised vital rates (e.g. $d=p$. $\left.d_{1}+(1-p) d_{2}\right)$

\section{Results}

\subsection{General behaviour}

The model exhibited a periodic steady state, with obvious gaps between the population sizes in favourable and unfavourable habitats (Fig. 1A). The gap size is more sensitive to changes in emigration probability $(d)$ than to changes in dispersal distance $\left(\sigma^{2}\right)$, with even higher sensitivity observed when increasing dispersal probability from favourable habitats than when increasing the same factor in unfavourable ones. When the proportion of favourable habitats $(p)$ increased, population sizes remained largely unchanged in favourable habitats whilst population sizes in unfavourable habitats increased notably. Similarly, increasing only the periodic length of spatial heterogeneity $(L)$ notably reduced the populations in unfavourable habitats.

Unless the population eventually became extinct, it was found to expand its range in both directions from the introduction location, in the form of a periodic travelling wave (i.e.u $\left(x+L ; t+t^{\prime}\right)=$ $u(x ; t)$ for some $t^{\prime}>0$ ) (Fig. 1A). (We note that Fig. 1 and the remaining figures in this section were obtained using Gaussian dispersal kernel) A time lag was often observed before the detection of the population after its initial introduction in an unfavourable patch (Fig. 1B). The time lag can be shortened by increasing the initial population size or the vital rates (growth and dispersal rates). Time lags on the other hand can be prolonged for larger thresholds of detection or wider unfavourable patches. Nonetheless, the spreading dynamics remained the same for populations in landscapes with different periodic lengths but a common proportion of favourable habitats $(p)$, regardless of whether it was initially introduced into a favourable or unfavourable patch. 


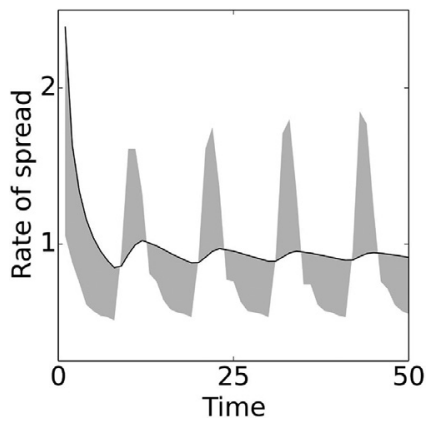

Fig. 2. Instantaneous rate and average rate of spread associated to a periodic length of habitat $L=10$. Other parameter values are $R_{1}=e, R_{2}=e^{-0.5}, d_{1}=0.75, d_{2}=1$, $\sigma_{1}^{2}=1, \sigma_{2}^{2}=1$. The instantaneous rates of spread are shown by the grey area.

As such, the following analysis was done for populations initially introduced in a favourable patch.

\subsection{Instantaneous and average rate of spread}

With the increase of periodic length $(L)$, the magnitude of fluctuation of the instantaneous rate of spread increased, although the average rate of spread was less sensitive to the changes of periodic length in the landscape (Fig. 2).

Let $M_{1}(\lambda)$ and $M_{2}(\lambda)$ denote the moment generating function of the dispersal kernels $k_{1}$ and $k_{2}$ respectively and

$M(\lambda)=\left(\begin{array}{cc}p d_{1} M_{1}(\lambda) R_{1}+\left(1-d_{1}\right) R_{1} & p d_{2} M_{2}(\lambda) R_{2} \\ (1-p) d_{1} M_{1}(\lambda) R_{1} & (1-p) d_{2} M_{2}(\lambda) R_{2}+\left(1-d_{2}\right) R_{2}\end{array}\right)$.

As derived in Appendix, the asymptotic rate can be approximated by

$c^{*}=\min _{\lambda} c(\lambda)$

where

$c(\lambda)=\frac{1}{\lambda} \log \left(\frac{1}{2}\left(\operatorname{Tr}(M(\lambda))+\sqrt{\operatorname{Tr}(M(\lambda))^{2}-4 \operatorname{Det}(M(\lambda))}\right)\right)$

and $\operatorname{Tr}(M(\lambda))$ and $\operatorname{Det}(M(\lambda))$ indicate the trace and determinant of the matrix $M(\lambda)$. In particular, when the emigration probabilities $d_{1}=d_{2}=1$, we obtain the relation established by Dewhirst and Lutscher (2009)

$c^{*}=\min _{\lambda} \frac{1}{\lambda} \ln \left(p M_{1}(\lambda) R_{1}+(1-p) M_{2}(\lambda) R_{2}\right)$.

The accuracy of the estimation in Eq. (2) is further supported by Fig. 3A, where the minimisation in Eq. (2) was performed using the function Minimize in Mathematica 10.0.

Understanding the dependence of the invasion conditions and the rate of spread on the vital rates can be challenging due to the nonlinear and implicit nature of Eq. (2). To obtain a more explicit dependence of the rate of spread on the vital rates, let

$$
\begin{aligned}
\hat{R} & =p d_{1} R_{1}+(1-p) d_{2} R_{2}+\left(1-d_{1}\right) R_{1}+\left(1-d_{2}\right) R_{2} \\
\tilde{R} & =\sqrt{\hat{R}^{2}-4\left(1-(1-p) d_{1}-p d_{2}\right) R_{1} R_{2}} \\
\bar{R} & =\frac{1}{2}(\hat{R}+\tilde{R}) \\
\bar{C}^{2} & =\frac{1}{2}\left(p d_{1} R_{1} \sigma_{1}^{2}+(1-p) d_{2} R_{2} \sigma_{2}^{2}\right) \\
\hat{C}^{2} & =2\left(p\left(1-d_{2}\right) d_{1} \sigma_{1}^{2}+(1-p)\left(1-d_{1}\right) d_{2} \sigma_{2}^{2}\right) R_{1} R_{2} \\
\tilde{C}^{2} & =2 \hat{R} \bar{C}^{2}+\hat{C}^{2}
\end{aligned}
$$

The rate of spread can be approximated (see Appendix) by

$c \approx \sqrt{2 \bar{C}^{2}+\frac{\tilde{C}^{2}}{\tilde{R}}} \sqrt{\frac{\log (\bar{R})}{\bar{R}}}$.

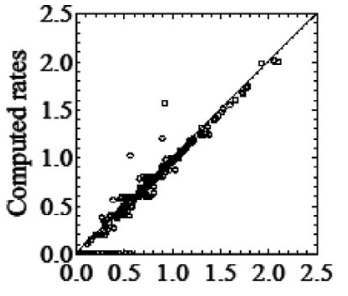

Eq. 2

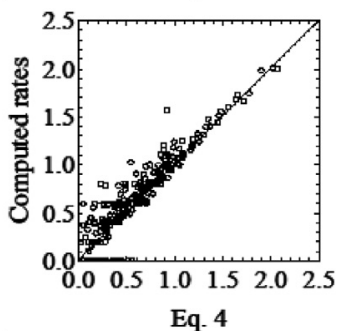

Fig. 3. Estimated and computed rate of spread for 300 set of parameter values. In the simulations, the period of environmental variation is $L=1$, the threshold of detection is 0.01 and local populations that fall below $10^{-16}$ were considered extinct. Other parameter values were randomly generated with the restrictions $0<p<1$, $1<R_{1}<2,0<R_{2}<1,0<d_{1}, d_{2}<1$ and $0<, \sigma_{1}^{2}, \sigma_{2}^{2}<5$.The points that lie on the $\mathrm{x}$-axis correspond to parameter values for which the population spreads but remained a low density (less than the threshold of detection).

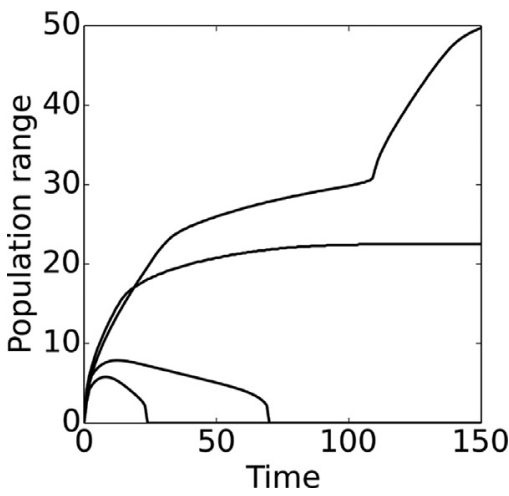

Fig. 4. The range front for given proportion of suitable habitat, $p=0.05,0.1$ (unsuccessful invasions), 0.5 and 0.75 (successful invasions). Parameter values are $R_{1}=e^{0.25}, R_{2}=e^{-0.25}, d_{1}=0.75, d_{2}=1, \sigma_{1}^{2}=1, \sigma_{2}^{2}=0.5$. The threshold of detection is $10^{-6}$.

Fig. 3 suggests that Eq. (4) provides a good approximation of the rate of spread derived in Eq. (2). Furthermore, the population can spread whenever $\bar{R}>1$ (Fig. 4) and the invasion condition can be reduced to

$p d_{1} R_{1}+\left(1-d_{1}\right) R_{1}+(1-p) d_{2} R_{2}+\left(1-d_{2}\right) R_{2}$

$-(1-(1-p) d 1-p d 2) R 1 R 2>1$.

With the increase of growth rates $\left(R_{1}\right.$ and $\left.R_{2}\right)$ and the variance of the dispersal kernels ( $\sigma_{1}^{2}$ and $\sigma_{2}^{2}$ ), the rate of spread will increase. However, the dependence of the rate of spread on the emigration probabilities $\left(d_{1}\right.$ and $\left.d_{2}\right)$ is less evident. We thus conducted a sensitivity test for the rate of spread as a function of $d_{1}$ and $d_{2}$ under different proportions of favourable habitats $p$ (Fig. 5). First, the rate of spread increased with the dispersal probability from unfavourable habitats $d_{2}$. Second, when $d_{1}<d_{1}^{*}$ (emigration probability in favourable habitats less than some threshold $d_{1}^{*}$ ), increasing $d_{1}$ will speed up the spread. Increasing $d_{1}$ beyond this threshold however could potentially slow down the spread or even results in the extinction of the population especially when the proportion of favourable habitat is low. High emigration from 


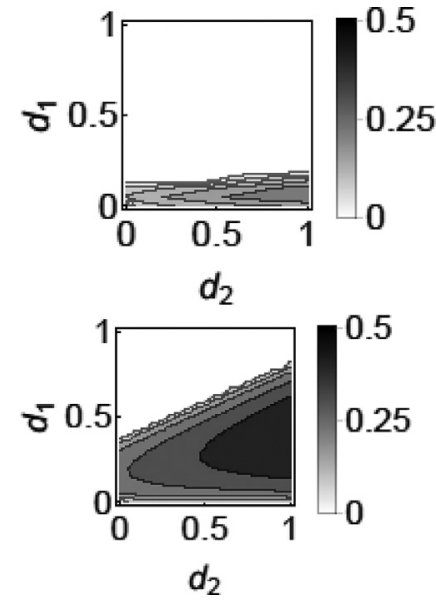

Fig. 5. The rate of spread as a function of the emigration probability $d_{1}$ from favourable habitats and the emigration probability $d_{2}$ from unfavourable habitats for (A) $p=0.25$ and (B) 0.75 . Other parameter values are $R_{1}=e^{0.1}, R_{2}=e^{-0.5}, \sigma_{1}^{2}=1$, $\sigma_{2}{ }^{2}=1$. The white area corresponds to parameter values for population extinction.

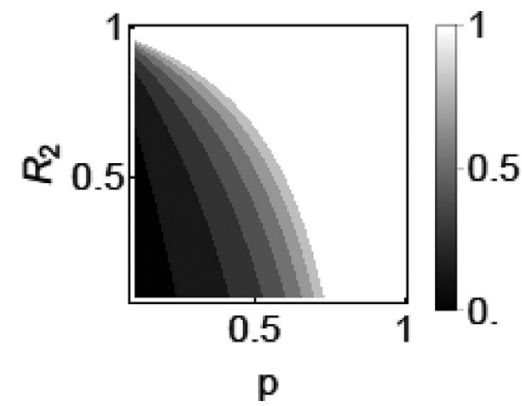

Fig. 6. The maximal emigration probability from favourable habitats that will lead to successful invasion as a function of the proportion of favourable habitats and the growth rate in unfavourable habitats. Other parameter values are $R_{1}=e^{0.1}$, $R_{2}=e^{-0.5}, \sigma_{1}^{2}=1, \sigma_{2}^{2}=1$ and $d_{2}=1$. The white area corresponds to parameter values for which the invasion will be successful regardless of the emigration from favourable habitats.

unfavourable habitats plus a moderate level of emigration from favourable habitats are the key to fast spread (Fig. 5).

The maximal emigration probability from favourable habitats that will lead to a successful spread can be obtained by solving Eq. (5) for $d_{1}$.Fig. 6 suggests that emigration from favourable habitats can lead of extinction of the population when the proportion of favourable habitat and the growth rates in unfavourable habitats are low.

Using the algorithm of Fang and Tacher [17] for generating landscapes with spatial autocorrelation $(I)$ ranging from 0 and 1 , we examined how the spatial arrangement of these favourable and unfavourable patches $\left(L_{1}=L_{2}=0.1\right)$ for a given proportion of favourable habitat $p$ affected the rate of spread. The mean asymptotic rate of spread from 15 simulations for each pair of $p$ and I showed no sensitivity to the change of spatial autocorrelation. However, the periodic length of the habitat $(L)$ for regular spacing did show a decline of spreading rate with increasing periodic length (Fig. 7).

\section{Discussion}

There have been different models that address the spread of species in spatially heterogeneous environments. In the case of continuous models using partial differential equations, Shigesada et al. [9] and Kinezaki et al. [10] have shown that the asymptotic rate of spread in a periodic patchy environment depends only on the space average growth rate and dispersal rate. Most spread

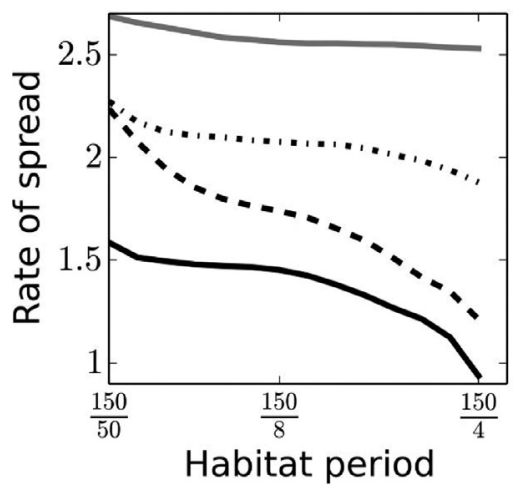

Fig. 7. The rate of spread as a function of the periodic length of alternating favourable and unfavourable patches. In the simulation, the total length of the habitat is 150 , and the periodic length $L$ varies from $1 / 50$ to $1 / 4$ of the total length at a step of 0.02 . Growth parameters are $R_{1}=e^{2}$ and $R_{2}=e^{-0.5}$. Black solid line: $p=0.5$, $d_{1}=0.5, d_{2}=1, \sigma_{1}^{2}=\sigma_{2}^{2}=1$. Dashed line: $p=0.5, d_{1}=0.5, d_{2}=1, \sigma_{1}^{2}=2, \sigma_{2}^{2}=1$. Dotted line: $p=0.5, d_{1}=0.5, d_{2}=1, \sigma_{1}^{2}=\sigma_{2}^{2}=2$. Grey line: $p=0.75, d_{1}=0.75$, $d_{2}=1, \sigma_{1}^{2}=\sigma_{2}^{2}=2$

models, in particular models in heterogeneous environments, have focused on different responses of growth and dispersal distances to habitat quality $[7,8]$. The influence of dispersal (emigration) probability in turn has received only little attention. Here, we have derived an approximation of the asymptotic rate of spread using the IDEs. Lower and upper bounds of the spreading rate (Eq. 2), as well as its dependence on the growth and dispersal parameters, are consistent with the results from [7] where only the growth rate varies spatially. It also corroborates with the results from [8] where both the growth rate and the dispersal kernel are locationdependent but with the dispersal probability $(d)$ being constant.

Habitat destruction normally includes two components, namely the overall loss of favourable habitats $(\mathrm{p} \downarrow)$ and the fragmentation of habitat patches $(\mathrm{L} \downarrow)[12,18,19]$. Previous studies suggest that the fate of many species is more sensitive to habitat loss. Although the habitat dependent emigration probability implies that part of the local populations may remain sedentary during the dispersal phase, these sedentary individuals can help to boost up the spread of the population. Especially when only a small proportion of the landscape is favourable for population growth (Fig. 5A), the rate of spread will be accelerated by having small emigration probability from favourable patches but decelerated by having a high emigration rate. When the proportion of favourable habitats is low, the majority of local populations are open to extinction. Emigrants from the rare favourable habitats therefore act to rescue these populations from extinction and thus facilitate the range expansion $[20,21]$.

Our results further confirmed Fahrig's speculation [14] that a high emigration probability does not always have a positive influence on the population dynamics (see also [22-24]). The rescue effect can sustain the range expansion only when the emigration probabilities from the favourable as well as unfavourable habitats are balanced $[25,26]$.When only a small proportion of the habitat is favourable for the growth, low emigration from favourable patches may not suffice to rescue populations in unfavourable patches from extinction. High emigration from favourable patches alone, on the other hand, can lead to the decline of rescuing individuals and yield a slower spread or even the extinction of the population following a boom-and-bust phenomenon. A minimal proportion of favourable habitat or maximal emigration from favourable habitats can be derived from the invasion condition (Eq. (6)) to ensure the persistence of the population. This invasion condition depends, not only on the growth parameters, but also the emigration probability of the population. The resulted boom-and-bust phenomenon is 
particularly important in invasion ecology, analogous to the concept of the rescue effect and extinction debt in the metapopulation literature $[21,25]$. Although local populations in unfavourable patches are doomed to extinction, the invasion can be successful as immigrants from favourable patches are likely to rescue populations in unfavourable patches from extinction (rescue effect).

In addition to the dependence on the availability of favourable habitats, the actual size of the favourable and unfavourable patches also affects the spread of a population [27,28]. Different studies have speculated a minimal patch size that constitutes an extinction threshold for an isolated population [29,30]. Our results further suggest that the spread of a population can decelerate with habitat fragmentation, more notably in species with lower emigration probability or dispersal distance (Fig. 7) [12,31]. This effect of habitat fragmentation has potentially prevented the effective control of pest species by their natural enemies $[32,33]$. In this context, the current study, in particular the approximation of the rate of spread can be used as a baseline to find the optimal management strategy of the level of habitat fragmentation as to promote the spread of the natural enemy for effectively controlling the pest population.

Finally, this work focused on the spread of a population in two types of habitats (which we referred to as favourable and unfavourable habitats). It is worth to note that the rate of spread in an environment with more habitat qualities could be derived by using the appropriate form of the matrix $M(\lambda)$. Namely, if the environment consists of alternating habitats characterised by $\left(R_{i}, d_{i}\right.$, $\sigma_{i}$ ) with proportion $p_{i}$ with $p_{1}+p_{2}+\cdots+p_{n}=1,(i=1,2, \ldots, n)$, the matrix $M(\lambda)$ is given by

$M(\lambda)=\left(\begin{array}{cc}p_{1} d_{1} M_{1}(\lambda) R_{1}+\left(1-d_{1}\right) R_{1} & p_{1} d_{2} M_{2}(\lambda) R_{2} \\ p_{2} d_{1} M_{1}(\lambda) R_{1} & p_{2} d_{2} M_{2}(\lambda) R_{2}+\left(1-d_{2}\right) R_{2} \\ \vdots & \\ p_{n} d_{1} M_{1}(\lambda) R_{1} & p_{n} d_{2} M_{2}(\lambda) R_{2}\end{array}\right.$

\section{Authors' contribution}

$\mathrm{AR}$ and $\mathrm{CH}$ proposed the research question and set up the model; AR conducted the analysis and simulation; both contributed to the writing.

\section{Acknowledgement}

We are grateful to Aziz Ouhinou and David Richardson for constructive discussion and Beverley Laniewski for English editing.

AR is supported by a Postdoctoral Fellowship from the African Institute for Mathematical Sciences. $\mathrm{CH}$ is the South African Research Chair in Mathematical and Theoretical Physical Biosciences, supported by the National Research Foundation (grants 89967 and 76912); he also receives support from the Australian Research Council (Discovery Project DP150103017).

\section{Appendix A. Derivation of the invasion condition and the asymptotic rate of spread}

The asymptotic rate of spread is derived hereafter. The main results are given in Eq. (A7) and Eq. (A9). First, we assume that the population is small at the front of the invasion and consider the linearisation of Eq. (1):

$$
\begin{aligned}
u(x ; t+1)= & \int[d(y) k(x-y ; y)+\delta(x, y)(1-d(y))] \\
& \times R(y) u(y ; t) d y .
\end{aligned}
$$

We recall that $g(u ; x) \leq R(x) u$; i.e. the population size is bounded by the linearisation. In what follows, we use heuristic methods to find an approximation of the rate of spread of the population governed by Eq. (A1).

Motivated by the periodicity of the growth and dispersal parameters on one hand, and numerical observations on the other, we assume that when the population does not eventually become extinct, it evolves into a travelling periodic wave (Kinezaki et al. 2006, See also Fig. 1B). In other words, there exists a number $t^{\prime}>$ osuch that $u\left(x+L ; t+t^{\prime}\right)=u(x ; t)$. The speed of the travelling periodic wave is defined by

$c=\frac{L}{t^{\prime}}$.

Here, we seek solutions of the form

$u(x ; t)=e^{\lambda(x+c(\lambda) t)} v(x)$,

for some $\lambda>0$, where $v$ is periodic in the space variable $(x)$ with the same period as the habitat $(v(x+L)=v(x))$ and $v(x) \geq 0$. Inserting Eq. (A3) into Eq. (A1) and using the fact that $d$, rand vare periodic, we have

$$
\begin{aligned}
e^{\lambda c(\lambda)} v(x)= & \sum_{n=-\infty}^{+\infty} \int_{0}^{L} d(y) k(x-y-n L, y) e^{\lambda(x-y-n L)} R(y) v(y) d y \\
& +(1-d(x)) R(x) v(x) .
\end{aligned}
$$

For exponentially bounded dispersal kernel, we can invert the order of the summation and integration and obtain the following,

$$
\begin{aligned}
& \left.\begin{array}{cc}
\cdots & p_{1} d_{n} M_{n}(\lambda) R_{n} \\
\ddots & \\
\cdots & p_{n} d_{n} M_{n}(\lambda) R_{n}+\left(1-d_{n}\right) R_{n}
\end{array}\right) \\
& e^{\lambda c(\lambda)} v(x)=\int_{0}^{L}\left[\sum_{n=-\infty}^{+\infty} k(x-y-n L, y) e^{\lambda(x-y-n L)}\right] \\
& \times d(y) v R(y)(y) d y+(1-d(x)) R(x) v(x) .
\end{aligned}
$$

For a sufficiently small $L$, we can use the approximation

$$
\sum_{n=-\infty}^{+\infty} k(x-y-n L, y) e^{\lambda(x-y-n L)}=\frac{1}{L} M(\lambda, y)
$$

when $x$ and $y$ are fixed, where $M$ is the moment generating function of the dispersal kernel at the location $y$ and is given by

$M(\lambda, y)=\int k(z, y) e^{\lambda z} d z$

We deduce that

$$
e^{\lambda c(\lambda)} v(x)=\frac{1}{L} \int_{0}^{L} M(\lambda, y) d(y) R(y) v(y) d y+(1-d(x)) R(x) v(x)
$$

for $0 \leq x \leq L$.

Integrating Eq. (A4) with respect to $x$ from 0 to $p L$ and from $p L$ to $L$ respectively we have

$$
\begin{aligned}
e^{\lambda c(\lambda)} \int_{0}^{p L} v(x) d x= & p L \frac{1}{L}\left[d_{1} M_{1}(\lambda) R_{1} \int_{0}^{p L} v(y) d y+d_{2} M_{2}(\lambda) R_{2} \int_{p L}^{L} v(y) d y\right] \\
& +\left(1-d_{1}\right) R_{1} \int_{0}^{p L} v(x) d x \\
e^{\lambda c(\lambda)} \int_{p L}^{L} v(x) d x= & (1-p) L \frac{1}{L}\left[d_{1} M_{1}(\lambda) R_{1} \int_{0}^{p L} v(y) d y+d_{2} M_{2}(\lambda) R_{2} \int_{p L}^{L} v(y) d y\right] \\
& +\left(1-d_{2}\right) R_{2} \int_{p L}^{L} v(x) d x
\end{aligned}
$$


By setting

$V_{1}=\int_{0}^{p L} v(x) d x$ and $V_{2}=\int_{p L}^{L} v(x) d x$

We have

$e^{\lambda c(\lambda)} V_{1}=p\left[d_{1} M_{1}(\lambda) R_{1} V_{1}+d_{2} M_{2}(\lambda) R_{2} V_{2}\right]+\left(1-d_{1}\right) R_{1} V_{1}$

$e^{\lambda c(\lambda)} V_{2}=(1-p)\left[d_{1} M_{1}(\lambda) R_{1} V_{1}+d_{2} M_{2}(\lambda) R_{2} V_{2}\right]+\left(1-d_{2}\right) R_{2} V_{2}$

From Eq. (A5), we deduce that $e^{\lambda c(\lambda)}$ is an eigenvalue of the matrix

$M=\left(\begin{array}{cc}p d_{1} M_{1}(\lambda) R_{1}+\left(1-d_{1}\right) R_{1} & p d_{2} M_{2}(\lambda) R_{2} \\ (1-p) d_{1} M_{1}(\lambda) R_{1} & (1-p) d_{2} M_{2}(\lambda) R_{2}+\left(1-d_{2}\right) R_{2}\end{array}\right)$,

associated with the positive eigenvector $\left(V_{1}, V_{2}\right)^{\mathrm{T}}$. By definition of the moment generating functions, the matrix $M$ is positive whenever the vital parameters are positive. We deduce using the Perron-Frobenius theorem and by noting that $\operatorname{Tr}(M)^{2}-4 \operatorname{Det}(M)>0$, that $e^{\lambda c(\lambda)}$ is the largest eigenvalue of the matrix $M$, that is

$e^{\lambda c(\lambda)}=\rho(\lambda)$, with $\rho(\lambda)=\frac{1}{2}\left(\operatorname{Tr}(M)+\sqrt{\operatorname{Tr}(M)^{2}-4 \operatorname{Det}(M)}\right)$

and

$c(\lambda)=\frac{1}{\lambda} \log [\rho(\lambda)]$.

Each wave shape $\lambda$ is associated with a solution of the form in Eq. (A3). The spread of the periodic travelling wave is given by

$c^{*}=\min _{\lambda} c(\lambda)$

For different dispersal kernels, the rate of spread can be obtained by solving the nonlinear system

$c^{*}=\frac{1}{\lambda^{*}} \log \left[\rho\left(\lambda^{*}\right)\right]$ and $c^{*}=\frac{\rho^{\prime}\left(\lambda^{*}\right)}{\rho\left(\lambda^{*}\right)}$

for the wave shape $\lambda^{*}$ and the corresponding wave speed $c^{*}$.

However, to have a more explicit dependence of the rate of spread on the vital rates, we consider the second order expansion of $\rho(\lambda)$ around 0 , and use the approximation of $c(\lambda)$

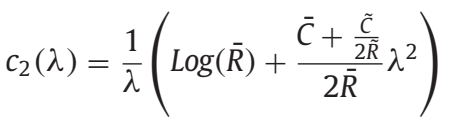

with

$$
\begin{aligned}
\hat{R} & =p d_{1} R_{1}+(1-p) d_{2} R_{2}+\left(1-d_{1}\right) R_{1}+\left(1-d_{2}\right) R_{2} \\
\tilde{R} & =\sqrt{\hat{R}^{2}-4\left(1-(1-p) d_{1}-p d_{2}\right) R_{1} R_{2}} \\
\bar{R} & =\frac{1}{2}(\hat{R}+\tilde{R}) \\
\bar{C}^{2} & =\frac{1}{2}\left(p d_{1} R_{1} \sigma_{1}^{2}+(1-p) d_{2} R_{2} \sigma_{2}^{2}\right) \\
\hat{C}^{2} & =2\left(p\left(1-d_{2}\right) d_{1} \sigma_{1}^{2}+(1-p)\left(1-d_{1}\right) d_{2} \sigma_{2}^{2}\right) R_{1} R_{2} \\
\tilde{C}^{2} & =2 \hat{R} \bar{C}^{2}+\hat{C}^{2}
\end{aligned}
$$

From Eq. (A8), the rate of spread can be approximated by

$c^{*} \approx \min _{\lambda} c_{2}(\lambda)=\sqrt{2 \bar{C}^{2}+\frac{\tilde{C}^{2}}{\tilde{R}}} \sqrt{\frac{\log (\bar{R})}{\bar{R}}}$.

\section{References}

[1] K.A. With, The landscape ecology of invasive spread, Conservation Biology 16 (5) (2002) 1192-1203.
[2] R. Aguilar, L. Ashworth, L. Galetto, M.A. Aizen, Plant reproductive susceptibility to habitat fragmentation: Review and synthesis through a meta-analysis, Ecol Lett 9 (2006) 968-980.

[3] C. Hui, R.M. Krug, D.M. Richardson, D.M. Richardson, Modelling spread in invasion ecology: A synthesis, Fifty Years of Invasion Ecology: The Legacy of Charles Elton, Wiley-Blackwell, Oxford, UK, 2010, doi:10.1002/9781444329988. ch25.

[4] A. Hastings, K. Cuddington, K.F. Davies, C.J. Dugaw, S. Elmendorf, A. Freestone, et al., The spatial spread of invasions: New developments in theory and evidence, Ecol. Lett. 4 (8) (2005) 91-101.

[5] C. Hui, N. Roura-Pascual, L. Brotons, R.A. Robinson, K.L. Evans, Flexible dispersal strategies in native and non-native ranges: environmental quality and the "good-stay, bad-disperse" rule, Ecography (Cop) 35 (2012) 1024-1032.

[6] H.F. Weinberger, K. Kawasaki, N. Shigesada, Spreading speeds of spatially periodic integro-difference models for populations with nonmonotone recruitment functions, J. Math. Biol. 57 (3) (2008) 387-411.

[7] K. Kawasaki, N. Shigesada, An integrodifference model for biological invasions in a periodically fragmented environment, Jpn . J. Ind. Appl. Math. 24 (1) (2007) 3-15

[8] S. Dewhirst, F. Lutscher, Dispersal in heterogeneous habitats: Thresholds, spatial scales, and approximate rates of spread. Ecology 90 (5) (2009) 1338-1345.

[9] N. Shigesada, K. Kawasaki, E. Teramoto, Traveling periodic waves in heterogeneous environments, Theor. Popul. Biol 30 (1) (1986) 143-160.

[10] N. Kinezaki, K. Kawasaki, N. Shigesada, Spatial dynamics of invasion in sinusoidally varying environments, Popul. Ecol. 48 (4) (2006) 263-270.

[11] M. Kot, M. Lewis, P. van den Driessche, Dispersal data and the spread of invading organisms, Ecology 77 (7) (1996) 2027-2042.

[12] M.a. Gilbert, S.M. White, J.M. Bullock, E.A. Gaffney, Spreading speeds for stage structured plant populations in fragmented landscapes, J. Theor. Biol. 30 (349) (2014) 135-149.

[13] R.H.G. Klaassen, B.A. Nolet, D. Bankert, Movement of foraging Tundra Swans explained by spatial pattern in cryptic food densities, Ecology 87 (2006) 2244-2254.

[14] L. Fahrig, Non-optimal animal movement in human-altered landscapes, Funct Ecol. 21 (6) (2007) 1003-1015.

[15] F. Lutscher, Density-dependent dispersal in integrodifference equations, J. Math. Biol 56 (4) (2008) 499-524.

[16] E. Jones, E. Oliphant, P. Peterson, et al., SciPy: Open source scientific tools for Python. http://www.scipy.org/, 2001 [Online; accessed 2015-06-02].

[17] J. Fang, L. Tacher, An efficient and accurate algorithm for generating spatially-correlated random fields, Commun. Numer. Meth. Engng. 19 (10) (2003) 801-808.

[18] L. Fahrig, Effects of habitat fragmentation on biodiversity, Annu. Rev. Ecol. Syst 34 (2003) 487-515.

[19] F.A. Bennett, D.A. Saunders, Habitat fragmentation and landscape change, Conservation Biology for all, Oxford University Press, 2010.

[20] A. Eriksson, F. Elías-Wolff, B. Mehlig, A. Manica, The emergence of the rescue effect from explicit within- and between-patch dynamics in a metapopulation, Proc. R. Soc. B (2014) 20133127.

[21] R. Etienne, Local populations of different sizes, mechanistic rescue effect and patch preference in the Levins metapopulation model, Bull. Math. Biol 62 (2000) 943-958.

[22] Y. Gruntfest, R. Arditi, Y. Domrovsky, A fragmented population in a varying environment, J. Theor. Biol 185 (1997) 539-547.

[23] R.W. Van Kirk, M.A. Lewis, Integrodifference models for persistence in fragmented habitats, Bull. Math. Biol. 59 (1) (1997) 107-137.

[24] A. Ramanantoanina, C. Hui, A. Ouhinou, Effects of density-dependent dispersa behaviours on the speed and spatial patterns of range expansion in predator-prey metapopulations, Ecol. Model. 222 (2011) 3524-3530.

[25] R. Levins, Some demographic and genetic consequences of environmental heterogeneity for biological control, Bull. Ecol. Soc. Am 15 (1969) 237-240.

[26] J. Horak, C. Hui, N. Roura-Pascual, D. Romportl, Changing roles of propagule, climate, and land use during extralimital colonization of a rose chafer beetle, Naturwissenschaften 100 (2013) 327-336.

[27] D. Bailey, M. Schmidt-Entling, P. Eberhart, J. Herrmann, G. Hofer, U. Kormann, et al., Effects of habitat amount and isolation on biodiversity in fragmented traditional orchards, J Appl. Ecol 47 (2010) 1003-1013.

[28] Z. Zhao, C. Hui, D. He, B. Li, Effects of agricultural intensification on ability of natural enemies to control aphids, Sci. Rep 5 (2014), doi:10.1038/srep08024.

[29] C.M. Taylor, A. Hastings, Allee effects in biological invasions, Ecol. Lett 8 (8) (2005) 895-908

[30] E.D. McCoy, H.R. Mushinsky, Estimates of minimum patch size depend on the method of estimation and the condition of the habitat, Ecology 88 (2007) 1401-1407.

[31] M. Gilbert, J. Grégoire, J.F. Freise, W. Heitland, Long-distance dispersal and human population density allow the prediction of invasive patterns in the horse chestnut leafminer Cameraria ohridella, Anim. Ecol 73 (2004) 459-468.

[32] K.A. With, Threshold effect of landscape structure on biological control in agrosystems, Ecol. Appl 12 (2002) 52-56.

[33] F. Ouyang C. Hui, S. Ge, X. Men, Z. Zhao, P. Shi, et al, Weakening density dependence from climate change and agricultural intensification triggers pest outbreaks: a 37-year observation of cotton bollworms, Ecol. Evol 4 (2014) 3362-3374. 